PROCEEDINGS OF THE

AMERICAN MATHEMATICAL SOCIETY

Volume 129, Number 2, Pages 467-474

S 0002-9939(00)05768-3

Article electronically published on August 28, 2000

\title{
UNIFORM ANTI-MAXIMUM PRINCIPLE FOR POLYHARMONIC BOUNDARY VALUE PROBLEMS
}

\author{
PHILIPPE CLÉMENT AND GUIDO SWEERS
}

(Communicated by David S. Tartakoff)

\begin{abstract}
A uniform anti-maximum principle is obtained for iterated polyharmonic Dirichlet problems. The main tool, combined with regularity results for weak solutions, is an estimate for positive functions in negative Sobolev norms.
\end{abstract}

\section{IntRoduCtion AND STATEMENT OF MAIN RESUlts}

Let us recall the situation for the second order boundary value problem

$$
\left\{\begin{aligned}
-\Delta u & =\lambda u+f & & \text { in } \Omega, \\
u & =0 & & \text { on } \partial \Omega,
\end{aligned}\right.
$$

where $\Omega$ is a bounded smooth domain in $\mathbb{R}^{n}$. It is well known that for $\lambda<\lambda_{1}$, with $\lambda_{1}$ the first eigenvalue, a sign preserving property holds: $f \geq 0$ implies that $u \geq 0$. In [4] it has been shown that when $\lambda-\lambda_{1}>0$ and small, a sign reversing phenomenon occurs. The more precise statement for (1.1) of this so-called antimaximum principle is as follows:

For $f \in L^{p}(\Omega)$ with $0 \neq f \geq 0$ and $p>n$ there exists $\lambda_{f}>\lambda_{1}$ such that for $\lambda \in\left(\lambda_{1}, \lambda_{f}\right)$ the solution $u$ of (1.1) satisfies $u<0$ in $\Omega$.

In [11] it has been proven that the restriction on $p$, that is $p>n$, is genuine. Indeed, for $\Omega$ smooth there is $f \in L^{n}(\Omega)$, with $f>0$, such that the solution $u$ of (1.1) changes sign for all $\lambda>\lambda_{1}$.

A much stronger result would have been that the sign reversing result holds for $\lambda \in\left(\lambda_{1}, \lambda_{1}+\delta\right)$ with $\delta>0$ independent of $f$. Such a result could be called a uniform anti-maximum principle and in fact exists for another boundary condition. Indeed for the one-dimensional Neumann problem, $-u^{\prime \prime}=\lambda u+f$ in $\Omega$ with $u^{\prime}=0$ on $\partial \Omega$; such a uniform anti-maximum principle was obtained in 4 .

In order to get a better understanding when the anti-maximum principle holds uniformly, we consider some elliptic systems of higher order: iterated polyharmonic Dirichlet problems. Let $m$ and $k$ be fixed positive integers. Defining

$$
\begin{aligned}
D(A) & =H^{2 m, 2}(\Omega) \cap H_{0}^{m, 2}(\Omega), \\
A & =(-\Delta)^{m}: D(A) \subset L^{2}(\Omega) \rightarrow L^{2}(\Omega),
\end{aligned}
$$

Received by the editors April 22, 1999.

1991 Mathematics Subject Classification. Primary 35J40, 35B50; Secondary 31B30.

Key words and phrases. Anti-maximum principle, higher order elliptic, polyharmonic.

(C)2000 American Mathematical Society 
we will study for $f \in L^{2}(\Omega)$ sign properties for $\lambda$ near $\lambda_{1}$ of

$$
A^{k} u=\lambda u+f,
$$

where $\lambda_{1}$ is the first eigenvalue of $A^{k}$. Equation (1.3) corresponds to the boundary value problem $(-\Delta)^{m k} u=\lambda u+f$ in $\Omega$, and $\left(\frac{\partial}{\partial n}\right)^{i}(-\Delta)^{m j} u=0$ on $\partial \Omega$ for $i=0, \ldots, m-1$ and $j=0, \ldots, k-1$. Here $n$ denotes the outward normal.

A necessary condition for sign-reversing and sign-preserving property for (1.3) to hold with $\lambda$ near $\lambda_{1}$, is that the first eigenvalue is simple and that the corresponding eigenfunction has a fixed sign. On general domains polyharmonic operators with Dirichlet boundary conditions do not have a first eigenfunction with a fixed sign. Boggio ([3]) however proved that for $\Omega=B$ a ball, the Green function for $A u=f$ is positive and hence, by results of Jentszch (8]) or Krein-Rutman (see [9]), the first eigenvalue is algebraically simple and has a strictly positive eigenfunction (see 7]). Although the eigenfunction remains positive under small perturbations of the domain ( $[7$ ) we will restrict ourselves to $\Omega=B$ when $m>1$. Note that eigenfunctions of $A$ and of $A^{m}, m>1$, coincide.

Theorem 1. Let $A$ be as in (1.2) and suppose that either

(i) $m=1$ and $\Omega$ is a bounded domain in $\mathbb{R}^{n}$ with $\partial \Omega \in C^{\infty}$, or

(ii) $m>1$ and $\Omega=\left\{x \in \mathbb{R}^{n} ;|x|<R\right\}$ for some $R>0$.

Let $\lambda_{1}$ be the smallest eigenvalue of $A^{k}$. If $n<2 m(k-1)$, then there exists $\delta>0$ such that for all $\lambda \in\left(\lambda_{1}, \lambda_{1}+\delta\right)$ and $f \in L^{2}(\Omega)$ with $0 \neq f \geq 0$ the solution $u$ of (1.3), which belongs to $C^{m}(\bar{\Omega})$, satisfies

$$
\left\{\begin{aligned}
u(x) & <0 \quad \text { for all } x \in \Omega, \\
\left(\frac{\partial}{\partial n}\right)^{i} u(x)=0 & \text { for all } x \in \partial \Omega \text { and } i \in\{0,1, \ldots, m-1\}, \\
\left(-\frac{\partial}{\partial n}\right)^{m} u(x) & <0 \quad \text { for all } x \in \partial \Omega .
\end{aligned}\right.
$$

The conditions of Theorem 1 guarantee that all eigenvalues are real and positive. Moreover, for $\lambda \in\left[0, \lambda_{1}\right)$ the system in (1.3) is sign preserving. See [7].

The inequality $n<2 m(k-1)$ is sharp. A proof of this fact will appear elsewhere.

The result in Theorem 1 coincides with that of Theorem 3 in 5 . In 5 more general, not necessarily self-adjoint, boundary value problems were considered using Sobolev spaces $H^{m, p}(\Omega)$ with $p \neq 2$. The non-Hilbert approach forces the proofs to be rather involved. An advantage of using $L^{p}$-type spaces is that one finds as an intermediate result a non-uniform anti-maximum principle. For the system in (1.3) it reads as:

Proposition 2. Let $A$ and $\Omega \subset \mathbb{R}^{n}$ be as in Theorem 1. If $n<2 p m\left(k-\frac{1}{2}\right)$, then for $f \in L^{p}(\Omega)$ with $0 \neq f \geq 0$ there exists a $\lambda_{f}>\lambda_{1}$ such that for all $\lambda \in\left(\lambda_{1}, \lambda_{f}\right)$ the solution $u$ of (1.3) which belongs to $C^{m}(\bar{\Omega})$ satisfies (1.4).

\section{Solutions to $A u=f$}

First we will recall and derive some properties of solutions to $A u=f$, that is, for the system

$$
\begin{cases}(-\Delta)^{m} u=f & \text { in } \Omega \\ u=\frac{\partial}{\partial n} u=\cdots=\left(\frac{\partial}{\partial n}\right)^{m-1} u=0 & \text { on } \partial \Omega\end{cases}
$$


The spaces $H^{k, 2}(\Omega)$ and $H_{0}^{k, 2}(\Omega)$ for $k \in \mathbb{Z}$ that we use are defined in [10]. We recall that

$$
H^{-s, 2}(\Omega)=\left(H_{0}^{s, 2}(\Omega)\right)^{\prime} \text { for } s \in \mathbb{R} .
$$

For short notation we set $H^{s, 2}=H^{s, 2}(\Omega)$, etc.

2.1. Strong solutions. It follows from Theorem 8.4 of [10, page 196] that the operator $A$ is self-adjoint in $L^{2}$. Indeed conditions (i), (ii) and (iii) of [10, page 148] are satisfied, the operator $A$ is formally self-adjoint and the boundary operators $C_{j}$ can be chosen equal to $B_{j}=\left(\frac{\partial}{\partial n}\right)^{j}$ in Theorem 8.4.

Next we show that $N(A)=\{0\}$. If $u \in D(A)$ satisfies $A u=0$, then $\langle\langle u, u\rangle\rangle_{m}=$ $\int_{\Omega} u A u d x=0$, where the bilinear form $\langle\langle\cdot, \cdot\rangle\rangle_{m}$ is defined by

$$
\langle\langle u, v\rangle\rangle_{m}= \begin{cases}\int_{\Omega} \Delta^{\frac{m}{2}} u \Delta^{\frac{m}{2}} v d x & \text { for } m \text { even, } \\ \int_{\Omega} \nabla\left(\Delta^{\frac{m-1}{2}} u\right) \cdot \nabla\left(\Delta^{\frac{m-1}{2}} v\right) d x & \text { for } m \text { odd. }\end{cases}
$$

By a one-dimensional version of an inequality of Poincaré it follows for $v \in H_{0}^{1,2}$ with $\Omega$ bounded, that

$$
\int_{\Omega} v^{2} d x \leq c_{\Omega} \int_{\Omega}\left(\frac{\partial v}{\partial x_{i}}\right)^{2} d x \text { for each } i \in\{1, \ldots, n\} .
$$

Hence there exists $c_{\Omega, m}>0$ such that

$$
\int_{\Omega} u^{2} d x \leq c_{\Omega, m}\langle\langle u, u\rangle\rangle_{m} \text { for all } u \in H_{0}^{m, 2} .
$$

Therefore $A u=0$ implies $u=0$. By applying Theorem 5.4 of [10, page 165] we obtain:

Lemma 1. Let $s \geq 0$. For each $f \in H^{s, 2}$ there exists a unique solution $u \in H_{0}^{m, 2} \cap$ $H^{2 m+s, 2}$ of 2.1. Moreover, there exists $c_{\Omega, m, s}>0$ such that

$$
\|u\|_{H^{2 m+s, 2}} \leq c_{\Omega, m, s}\|f\|_{H^{s, 2}} \text { for all } f \in H^{s, 2} .
$$

2.2. Weak solutions. By duality we may extend the estimate in (2.6) for $s<0$. Note that [10. Theorem 8.3, page 195] extends the estimate in (2.6) to $s \in[-m, 0$ ) with $s+\frac{1}{2} \notin \mathbb{Z}$. For $s \in[-2 m,-m)$ the same estimate is no longer true due to the fact that $H^{s, 2}=\left(H_{0}^{-s, 2}\right)^{\prime}$ has to be replaced with $\left(H_{0}^{m, 2} \cap H^{-s, 2}\right)^{\prime}$. For sake of short notation we will use for $\kappa \in \mathbb{N}$

$$
\begin{gathered}
\|f\|_{-m,-\kappa}=\|f\|_{\left(H_{0}^{m, 2} \cap H^{m+\kappa, 2}\right)^{\prime}} \\
=\sup \left\{|\langle\varphi, f\rangle| ; \varphi \in H_{0}^{m, 2} \cap H^{m+\kappa, 2} \text { with }\|\varphi\|_{H^{m+\kappa, 2}} \leq 1\right\},
\end{gathered}
$$

where $\langle\varphi, f\rangle$ denotes the value of the functional $f$ at $\varphi$. For $f, g \in L^{2}$ we will also use the notation $\langle f, g\rangle=\int_{\Omega} f g d x$.

Notice that for $m, \kappa>0$ the norm $\|\cdot\|_{-m-\kappa, 0}\left(\right.$ resp. $\left.\|\cdot\|_{-m,-\kappa}\right)$ is strictly weaker than the norm $\|\cdot\|_{-m,-\kappa}$ (resp. $\|\cdot\|_{0,-m-\kappa}$ ).

According to Lemma 1 the operator $A_{0}: H_{0}^{m, 2} \cap H^{2 m, 2} \rightarrow L^{2}$ defined by $A_{0} u=$ $A u$ is an isomorphism. Hence $A_{-1}: L^{2} \rightarrow\left(H_{0}^{m, 2} \cap H^{2 m, 2}\right)^{\prime}$ defined by $A_{-1}=A_{0}^{\prime}$ is an isomorphism, that is, for all $v \in L^{2}$ there is unique $f \in\left(H_{0}^{m, 2} \cap H^{2 m, 2}\right)^{\prime}$ with $A_{-1} v=f$ in the sense that $\int_{\Omega} v A \varphi d x=\langle\varphi, f\rangle$ for all $\varphi \in H_{0}^{m, 2} \cap H^{2 m, 2}$. From the 
self-adjointness of $A$ it follows that $A_{-1}$ is an extension of $A_{0}$. We get the following scheme:

$$
H_{0}^{m, 2} \cap H^{2 m, 2} \stackrel{A_{0}}{\hookrightarrow} L^{2} \simeq\left(L^{2}\right)^{\prime} \stackrel{A_{-1}}{\hookrightarrow}\left(H_{0}^{m, 2} \cap H^{2 m, 2}\right)^{\prime} .
$$

Defining for $\kappa \in \mathbb{N}^{+}$the spaces

$$
\begin{aligned}
B_{\kappa} & =\left\{u \in H^{2 m \kappa, 2} ; A^{j} u \in H_{0}^{m, 2} \text { for } 0 \leq j \leq \kappa-1\right\}, \\
B_{-\kappa} & =\left(B_{\kappa}\right)^{\prime}
\end{aligned}
$$

and $B_{0}=L^{2} \simeq\left(L^{2}\right)^{\prime}$, we obtain the following scale of Hilbert spaces with restrictions and extensions of $A_{0}$ :

$$
\ldots \stackrel{A_{\kappa}}{\hookrightarrow} B_{\kappa} \stackrel{A_{\kappa-1}}{\hookrightarrow} \ldots \frac{A_{1}}{\hookrightarrow} B_{1} \underset{\hookrightarrow}{\stackrel{A_{0}}{\hookrightarrow}} B_{0} \underset{\hookrightarrow}{\stackrel{A_{-1}}{\hookrightarrow}} B_{-1} \underset{\hookrightarrow}{\stackrel{A_{-2}}{\hookrightarrow}} \ldots \underset{\hookrightarrow}{\stackrel{A_{-\kappa}}{\hookrightarrow}} B_{-\kappa} \stackrel{A_{-\kappa-1}}{\hookrightarrow} \ldots
$$

See [2, Chapter V]. The operators $A_{\kappa}: B_{\kappa+1} \rightarrow B_{\kappa}$ for $\kappa \in \mathbb{Z}$ are isomorphisms. Finally, we introduce the (complex) interpolation spaces $B_{\kappa+\frac{1}{2}}$ defined by

$$
B_{\kappa+\frac{1}{2}}=\left[B_{\kappa}, B_{\kappa+1}\right]_{\frac{1}{2}} .
$$

Lemma 2. Let $\kappa \in \mathbb{Z}$.

(i) If $\kappa \geq 0$, then $B_{\kappa+\frac{1}{2}}=\left\{u \in H^{2 m \kappa+m, 2} ; A^{j} u \in H_{0}^{m, 2}\right.$ for $\left.0 \leq j \leq \kappa\right\}$.

(ii) If $\kappa<0$, then $B_{\kappa+\frac{1}{2}}=\left(B_{-\kappa-\frac{1}{2}}\right)^{\prime}$.

Proof. It is known (see [13. Thm 4.3.3, p.321]) that for $\kappa>0$ the space $\left[B_{\kappa}, B_{\kappa+1}\right]_{1 / 2}$ is the subspace of $H^{2 m \kappa+m, 2}=\left[H^{2 m(\kappa+1), 2}, H^{2 m \kappa, 2}\right]_{1 / 2}$ submitted to exactly all boundary conditions of $B_{\kappa+1}$ that are of order less then $2 m \kappa+m-\frac{1}{2}$. The second result follows from $\left[X^{\prime}, Y^{\prime}\right]_{\theta}=[X, Y]_{\theta}^{\prime}$ (see [10, Thm 6.2, p.29]).

\section{Positivity AND Simplicity of the First EIgENFunCtion}

We will need the first eigenvalue to be simple, with the corresponding eigenfunction positive and having an appropriate behavior at the boundary.

Let $d$ denote the distance to $\partial \Omega$ :

$$
d(x, \partial \Omega)=\inf \{|x-y| ; y \in \partial \Omega\} .
$$

Lemma 3. Let $\Omega$ be as in Theorem 1. Then the first eigenvalue $\mu_{m, 1}$ of $A$ is strictly positive and simple. Moreover, the corresponding eigenfunction $\varphi_{m, 1}$, chosen positive, satisfies for some $c_{m}, C_{m}>0$

$$
c_{m} d(x, \partial \Omega)^{m} \leq \varphi_{m, 1}(x) \leq C_{m} d(x, \partial \Omega)^{m} \text { for all } x \in \Omega .
$$

Proof. If $m=1$ and $\Omega$ is a bounded domain in $\mathbb{R}^{n}$ with smooth boundary, the eigenfunction for the first eigenvalue is positive. The estimate in (3.2) follows from Hopf's boundary point lemma and $\varphi_{1,1} \in C^{1}(\bar{\Omega}) \cap C_{0}(\bar{\Omega})$.

Now suppose that $m>1$. Then $\Omega=\left\{x \in \mathbb{R}^{n} ;|x|<R\right\}$ and the explicit formula of the Green function by Boggio [3, (48), page 126] guarantees that the solution $u$ of $(-\Delta)^{m} u=f \in C(\bar{\Omega})$ with $0 \neq f \geq 0$ with the Dirichlet boundary conditions $\left(\frac{\partial}{\partial n}\right)^{j} u=0$ on $\partial \Omega, j=0, \ldots, m-1$, satisfies for some $c_{f}>0$

$$
u(x) \geq c_{f} d(x, \partial \Omega)^{m} \text { for all } x \in \Omega .
$$


Since the Green function is positive Jentszch' Theorem ([8]), or Krein-Rutman, implies that the first eigenvalue $\lambda_{m, 1}$ is algebraically simple and that the corresponding eigenfunction $\varphi_{m, 1}$ is positive. Using (3.3) and the fact that $\varphi_{m, 1} \in$ $C^{m}(\bar{\Omega}) \cap C_{0}^{m-1}(\bar{\Omega})$ there are $c_{m}, C_{m}>0$ such that

$$
c_{m} d(x, \partial \Omega)^{m} \leq \varphi_{m, 1}(x) \leq C_{m} d(x, \partial \Omega)^{m} \text { for all } x \in \Omega,
$$

which completes the proof.

\section{Solving By EIGENFUnCtions}

Recall that the unbounded operator $A: D(A) \subset L^{2} \rightarrow L^{2}$ is positive self-adjoint (see 2.5). Since the imbedding of $H^{2 m, 2}$ in $L^{2}$ is compact we find that $A^{-1}: L^{2} \rightarrow$ $L^{2}$ is compact and positive symmetric. Hence $L^{2}$ has a complete orthonormal system consisting of eigenfunctions of $A$. Let us denote these eigenfunctions by $\left\{\varphi_{m, i}\right\}_{i=1}^{\infty}$ and the corresponding eigenvalues by $\left\{\mu_{m, i}\right\}_{i=1}^{\infty}$, that is, for $i, j \in \mathbb{N}^{+}$

$$
\left\{\begin{aligned}
(-\Delta)^{m} \varphi_{m, i} & =\mu_{m, i} \varphi_{m, i} & & \text { in } \Omega, \\
\left(\frac{\partial}{\partial n}\right)^{\kappa} \varphi_{m, i} & =0 & & \text { on } \partial \Omega \text { for } \kappa=0,1, \ldots, m, \\
\left\langle\varphi_{m, i}, \varphi_{m, j}\right\rangle & =\delta_{i j} . & &
\end{aligned}\right.
$$

Using (2.6) repeatedly one finds that $\varphi_{m, i} \in H^{k, 2}$ for all $k \in \mathbb{N}$ and hence $\varphi_{m, i} \in$ $C^{\infty}(\bar{\Omega})$. An equivalent norm on the space $B_{\kappa}$ defined in (2.9 2.11) for $\kappa \in \frac{1}{2} \mathbb{Z}$ is given by

$$
\|u\|_{B_{\kappa}}=\left(\sum_{i=1}^{\infty} \mu_{m, i}^{2 \kappa}\left\langle\varphi_{m, i}, u\right\rangle^{2}\right)^{\frac{1}{2}}
$$

We may use these eigenfunctions to solve (1.3). Note that $\lambda_{1}=\mu_{m, 1}^{k}$.

Lemma 4. There exist $C_{k, m, \Omega}>0$ and $\delta>0$ such that the following holds. Let $\lambda \in \mathbb{R}$ with $\left|\lambda-\mu_{m, 1}^{k}\right|<\delta$. For all $f \in B_{-\frac{1}{2} k}$ with $\left\langle\varphi_{m, 1}, f\right\rangle=0$ there exists a unique weak solution $u_{\lambda} \in B_{\frac{1}{2} k}$ to

$$
\left\{\begin{array}{c}
\left(A^{k}-\lambda\right) u=f \\
\left\langle\varphi_{m, 1}, u\right\rangle=0
\end{array}\right.
$$

and moreover

$$
\left\|u_{\lambda}\right\|_{B_{\frac{1}{2} k}} \leq C_{k, m, \Omega}\|f\|_{B_{-\frac{1}{2} k}} .
$$

Proof. The lemma is an immediate consequence of $\left\langle\varphi_{m, 1}, f\right\rangle=0$, the solution formula

$$
u_{\lambda}=\sum_{i=2}^{\infty} \frac{1}{\mu_{m, i}^{k}-\lambda}\left\langle\varphi_{m, i}, f\right\rangle \varphi_{m, i}
$$

and choosing $\delta \in\left(0, \mu_{m, 2}^{k}-\mu_{m, 1}^{k}\right)$. 


\section{A weighted $C$-SPACE}

Let us define $C_{d^{m}}(\bar{\Omega})=\left\{u \in C(\bar{\Omega}) ;\|u\|_{d^{m}}<\infty\right\}$ where

$$
\|u\|_{d^{m}}=\sup \left\{\left|\frac{u(x)}{d(x, \partial \Omega)^{m}}\right| ; x \in \Omega\right\} .
$$

A similar space $C_{e}(\bar{\Omega})$ has been used by Amann in [1], where $e$ is the solution to $-\Delta e=1$ in $\Omega$ and $e=0$ on $\partial \Omega$. For $\partial \Omega \in C^{1, \gamma}$ the spaces $C_{e}(\bar{\Omega})$ and $C_{d^{1}}(\bar{\Omega})$ coincide. Note that

$$
C_{0}^{m-1}(\bar{\Omega}) \cap C^{m}(\bar{\Omega}) \hookrightarrow C_{d^{m}}(\bar{\Omega}) \hookrightarrow C_{0}(\bar{\Omega}) .
$$

5.1. An imbedding. The next lemma is a consequence of the imbedding

$$
H_{0}^{m} \cap H^{m+\kappa} \hookrightarrow C_{0}^{m-1}(\bar{\Omega}) \cap C^{m}(\bar{\Omega}) \text { for } n<2 \kappa .
$$

Lemma 5. Let $\Omega \subset \mathbb{R}^{n}$ be a bounded domain with $\partial \Omega \in C^{\infty}$ and suppose that $n<2 \kappa$. Then there exists $c_{\Omega, m, \kappa}>0$ such that for all $u \in H_{0}^{m} \cap H^{m+\kappa}$

$$
\|u\|_{d^{m}} \leq c_{\Omega, m, \kappa}\|u\|_{H^{m+\kappa}} .
$$

Proof. Since $2 \kappa>n$ the Rellich-Kondrachov Theorem shows that there exists a $c_{\Omega, \kappa}>0$ such that $\|u\|_{C^{m}(\bar{\Omega})} \leq c_{\Omega, \kappa}\|u\|_{H^{m+\kappa}}$. If $v \in C^{1}(\bar{\Omega}) \cap H_{0}^{1}$, then $v=0$ on $\partial \Omega$ and hence (an inequality of Poincaré)

$$
|v(x)| \leq\|v\|_{C^{1}(\bar{\Omega})} d(x, \partial \Omega) \text { for all } x \in \Omega .
$$

A repeated use of the last inequality shows that $u \in C^{m}(\bar{\Omega}) \cap H_{0}^{m}$ satisfies

$$
|u(x)| \leq \frac{1}{m !}\|u\|_{C^{m}(\bar{\Omega})} d(x, \partial \Omega)^{m} \text { for all } x \in \Omega,
$$

and hence (5.4) follows with $c_{\Omega, m, \kappa}=c_{\Omega, \kappa} / m !$.

By the previous lemma and the definition of the norm in (2.7) for the dual space we find:

Corollary 3. Let $\Omega \subset \mathbb{R}^{n}$ be a bounded domain with $\partial \Omega \in C^{\infty}$ and suppose that $n<2 \kappa$. Then there exists $c_{\Omega, m, \kappa}>0$ such that for all $f \in L^{2}$

$$
\|f\|_{-m,-\kappa} \leq c_{\Omega, m, \kappa} \sup \left\{|\langle\varphi, f\rangle| ; \varphi \in C_{d^{m}}(\bar{\Omega}) \text { with }\|\varphi\|_{d^{m}} \leq 1\right\} .
$$

\section{An estimate for positive functions in a negative Sobolev SPaCe}

Proposition 4. Let $\varphi_{m, 1}$ be the first eigenfunction of (4.1) satisfying (3.4) and normalized by $\left\langle\varphi_{m, 1}, \varphi_{m, 1}\right\rangle=1$. Let $2 \kappa>n$. Then there exists $c>0$ such that for all $f \in L^{2}$ with $f \geq 0$ the following estimate holds:

$$
\left\|f-\left\langle\varphi_{m, 1}, f\right\rangle \varphi_{m, 1}\right\|_{-m,-\kappa} \leq c\left\langle\varphi_{m, 1}, f\right\rangle .
$$

Proof. Let us define $f_{e}=f-\left\langle\varphi_{m, 1}, f\right\rangle \varphi_{m, 1}$. In view of (5.7) it is sufficient to prove that there exists a constant $C$ such that

$$
\left\langle\varphi, f_{e}\right\rangle \leq C\left\langle\varphi_{m, 1}, f\right\rangle
$$

for all $\varphi \in C_{d^{m}}(\bar{\Omega})$ with $\|\varphi\|_{d^{m}} \leq 1$.

Let $\varphi$ be such a function. Hence by (3.2) we have

$$
|\varphi(x)| \leq c_{m}^{-1} \varphi_{m, 1}(x) \text { for all } x \in \Omega \text {. }
$$


Since $\left\langle\varphi_{m, 1}, f_{e}\right\rangle=0$ it follows that

$$
\left\langle\varphi, f_{e}\right\rangle=\left\langle c_{m}^{-1} \varphi_{m, 1}-\varphi,-f_{e}\right\rangle
$$

where (6.3) shows that $c_{m}^{-1} \varphi_{m, 1}-\varphi \geq 0$. From $f \geq 0$ we obtain $-f_{e} \leq\left\langle\varphi_{m, 1}, f\right\rangle \varphi_{m, 1}$ and hence

$$
\left\langle c_{m}^{-1} \varphi_{m, 1}-\varphi,-f_{e}\right\rangle \leq\left\langle c_{m}^{-1} \varphi_{m, 1}-\varphi,\left\langle\varphi_{m, 1}, f\right\rangle \varphi_{m, 1}\right\rangle .
$$

Since $\left\langle\varphi_{m, 1}, f\right\rangle \varphi_{m, 1} \geq 0$ and since (6.3) also implies that $c_{m}^{-1} \varphi_{m, 1}+\varphi \geq 0$, we find

$$
\left\langle c_{m}^{-1} \varphi_{m, 1}-\varphi,\left\langle\varphi_{m, 1}, f\right\rangle \varphi_{m, 1}\right\rangle \leq\left\langle 2 c_{m}^{-1} \varphi_{m, 1},\left\langle\varphi_{m, 1}, f\right\rangle \varphi_{m, 1}\right\rangle \text {. }
$$

Combining (6.4), 6.5, 6.6) and $\left\langle\varphi_{m, 1}, \varphi_{m, 1}\right\rangle=1$ we obtain (6.2) with $C=2 c_{m}^{-1}$.

Proposition 4 can be reformulated as an imbedding of $L^{1}\left(\Omega, \varphi_{m, 1} d x\right)$ into $\left(H_{0}^{m, 2} \cap H^{m+\kappa, 2}\right)^{\prime}$.

Corollary 5. Let $\varphi_{m, 1}$ and $\kappa$ be as in Proposition 4. Then there exists $c_{1}>0$ such that

$$
\|f\|_{-m,-\kappa} \leq c_{1} \int_{\Omega}|f| \varphi_{m, 1} d x \quad \text { for all } f \in L^{1}\left(\Omega, \varphi_{m, 1} d x\right) .
$$

Proof. Since $L^{2}$ is dense in $L^{1}\left(\Omega, \varphi_{m, 1} d x\right)$ it is sufficient to show (6.7) for $f \in L^{2}$. Set $f^{+}=\frac{1}{2}(|f|+f)$. By Proposition 4 we find

$$
\begin{aligned}
\left\|f^{+}\right\|_{-m,-\kappa} & \leq\left\|f^{+}-\left\langle\varphi_{m, 1}, f^{+}\right\rangle \varphi_{m, 1}\right\|_{-m,-\kappa}+\left\|\left\langle\varphi_{m, 1}, f^{+}\right\rangle \varphi_{m, 1}\right\|_{-m,-\kappa} \\
& \leq\left(c+c_{\varphi_{m, 1}}\right) \int_{\Omega} f^{+} \varphi_{m, 1} d x .
\end{aligned}
$$

With the estimate for $f^{-}=\frac{1}{2}(|f|-f)$ we find (6.7) for $c_{1}=c+c_{\varphi_{m, 1}}$.

\section{Proof of the MAIN Result}

In order to prove Theorem 1 we consider $S_{\lambda}: B_{\frac{1}{2} k} \rightarrow B_{-\frac{1}{2} k}$ defined by $S_{\lambda}=$ $A^{k}-\lambda$. For $f \in B_{-\frac{1}{2} k}$ and $\lambda \neq \mu_{m, i}^{k}=\lambda_{1}$ the solution $u$ satisfies

$$
u=\frac{1}{\mu_{m, 1}^{k}-\lambda}\left\langle\varphi_{m, 1}, f\right\rangle \varphi_{m, 1}+u_{e, \lambda}
$$

where $u_{e, \lambda}=\left(S_{\lambda}\right)^{-1} f_{e}$ with $f_{e}=f-\left\langle\varphi_{m, 1}, f\right\rangle \varphi_{m, 1}$. By Lemma 4 we have

$$
\left\|u_{e, \lambda}\right\|_{B_{\frac{1}{2} k}} \leq C_{1}\left\|f_{e}\right\|_{B_{-\frac{1}{2} k}}
$$

and since $B_{-\frac{1}{2} k} \supset\left(H_{0}^{m} \cap H^{k m}\right)^{\prime}$ it follows that

$$
\left\|f_{e}\right\|_{B_{-\frac{1}{2} k}} \leq C_{2}\left\|f_{e}\right\|_{-m,-(k-1) m} .
$$

By Proposition 4 we find if $2(k-1) m>n$ that

$$
\left\|f_{e}\right\|_{-m,-(k-1) m} \leq C_{3}\left\langle\varphi_{m, 1}, f\right\rangle .
$$

Since $u_{e, \lambda} \in B_{\frac{1}{2} k} \subset H_{0}^{m} \cap H^{k m}$ we have

$$
\left\|u_{e, \lambda}\right\|_{H_{0}^{m} \cap H^{k m}} \leq C_{4}\left\|u_{e, \lambda}\right\|_{B_{\frac{1}{2} k}}
$$


and assuming $2(k-1) m>n$ it follows by Lemma 5 with $\kappa=(k-1) m$ that

$$
\left\|u_{e, \lambda}\right\|_{d^{m}} \leq C_{5}\left\|u_{e, \lambda}\right\|_{H_{0}^{m} \cap H^{k m}} .
$$

Hence for all $\lambda$ with $\left|\lambda-\mu_{m, 1}^{k}\right|<\delta$ and $\delta$ as in Lemma 4 we find by using (17.6), (7.5),$(7.2)$, (7.3) , and (7.4):

$$
\left|u_{e, \lambda}(x)\right| \leq C_{6}\left\langle\varphi_{m, 1}, f\right\rangle d(x, \partial \Omega)^{m} .
$$

Finally, using the estimate in Lemma 3 for $\varphi_{m, 1}$ we find that if $0<\lambda-\mu_{m, 1}^{k}<\delta_{1}$ with $\delta_{1}=\min \left\{\delta, \frac{c_{m}}{C_{6}}\right\}$, then

$$
u(x) \leq\left(\frac{1}{\mu_{m, 1}^{k}-\lambda}\left\langle\varphi_{m, 1}, f\right\rangle+\frac{C_{6}}{c_{m}}\left\langle\varphi_{m, 1}, f\right\rangle\right) \varphi_{m, 1}(x)<0 .
$$

Since $-\varphi_{m, 1}$ satisfies the estimates in (1.4) the solution $u$ satisfies these same estimates.

Observe that for $-\delta_{1}<\lambda-\mu_{m, 1}^{k}<0$ a similar estimate from below recovers the maximum principle.

\section{REFERENCES}

[1] Amann, H., Fixed point equations and nonlinear eigenvalue problems in ordered Banach spaces, SIAM Rev. 18 (1976), no. 4, 620-709. MR 57:7269

[2] Amann, H., Linear and quasilinear parabolic problems, Vol. I. Abstract linear theory, Monographs in Mathematics 89, Birkhäuser Verlag, Basel, 1995. MR 96g:34088

[3] Boggio, T., Sulle funzioni di Green d'ordine m, Rend. Circ. Mat. Palermo 20 (1905), 97-135.

[4] Clément, Ph., and Peletier, L.A., An anti-maximum principle for second order elliptic operators, J. Differ. Equations 34 (1979), 218-229. MR 83c:35034

[5] Clément, Ph., and Sweers, G., Uniform anti-maximum principles, to appear in J. Differ. Equations.

[6] Grunau, H.-Ch. and Sweers, G., Positivity for equations involving polyharmonic elliptic operators with Dirichlet boundary conditions, Math. Ann. 307 (1997), 589-626. MR 98g:35058

[7] Grunau, H.-Ch. and Sweers, G., The maximum principle and positive principal eigenfunctions for polyharmonic equations, in Reaction Diffusion systems, Marcel Dekker Inc., New York 1997, p 163-182. MR 98h:35050

[8] Jentszch, P., Über Integralgleichungen mit positivem Kern, J. Reine Angew. Math. 141 (1912), 235-244.

[9] Krasnosel'skij, M.A., Lifshits, Je.A., and Sobolev, A.V., "Positive Linear Systems", Heldermann Verlag, Berlin, 1989. MR 91f:47051

[10] Lions, J.L. and Magenes, E., "Non-homogeneous Boundary Value Problems and Applications I", Springer, Berlin, 1972. MR 50:2670

[11] Sweers, G., $L^{N}$ is sharp for the antimaximum principle, J. Differential Equations 134 (1997), 148-153. MR 98a:35011

[12] Takáč, P., An abstract form of maximum and anti-maximum principles of Hopf's type, J. Math. Anal. Appl. 201 (1996), no. 2, 339-364. MR 97c:35016

[13] Triebel, H., "Interpolation Theory, Function Spaces, Differential Operators", North-Holland, Amsterdam, 1978. MR 80i:46032b

Department of Mathematics, Delft University of Technology, Mekelweg 4, 2628 CD Delft, The Netherlands

E-mail address: clement@twi.tudelft.nl

URL: http://aw.twi.tudelft.nl/ clement/

Department of Mathematics, Delft University of Technology, Mekelweg 4, 2628 CD

Delft, The Netherlands

E-mail address: sweers@twi.tudelft.nl

URL: http://aw.twi.tudelft.nl/ ${ }^{\text {sweers/ }}$ 